\title{
The National Ignition Facility: an experimental platform for studying behavior of matter under extreme conditions
}

\author{
Edward Moses
}

Received: 24 May 2010 / Accepted: 6 November 2010 / Published online: 24 November 2010

(C) The Author(s) 2010. This article is published with open access at Springerlink.com

\begin{abstract}
The National Ignition Facility (NIF), a 192-beam Nd-glass laser facility capable of producing $1.8 \mathrm{MJ}$ and $500 \mathrm{TW}$ of ultraviolet light, is now operational at Lawrence Livermore National Laboratory (LLNL). As the world's largest and most energetic laser system, NIF serves as the national center for the U.S. Department of Energy (DOE) and National Nuclear Security Administration to achieve thermonuclear burn in the laboratory and to explore the behavior of matter at extreme temperatures and energy densities. By concentrating the energy from all of its 192 extremely energetic laser beams into a $\mathrm{mm}^{3}$-sized target, NIF can reach the conditions required to initiate fusion reactions. NIF can also provide access to extreme scientific environments: temperatures about 100 million $\mathrm{K}$, densities of $1,000 \mathrm{~g} / \mathrm{cm}^{3}$, and pressures 100 billion times atmospheric pressure. These conditions have never been created before in a laboratory and exist naturally only in interiors of the planetary and stellar environments as well as in nuclear weapons.
\end{abstract}

Since August 2009, the NIF team has been conducting experiments in support of the National Ignition Campaign (NIC) - a partnership among LLNL, Los Alamos National Laboratory, General Atomics, the University of Rochester, Sandia National Laboratories, as well as a number of universities and international collaborators. The results from these initial experiments show promise for the relatively near-term achievement of ignition. Capsule implosion experiments at energies up to 1.2 MJ have demonstrated laser energetics, radiation temperatures, and symmetry control

\section{E. Moses $(\bowtie)$}

National Ignition Facility and Photon Science, Lawrence Livermore National Laboratory, 7000 East Avenue, Livermore, CA 94551, USA

e-mail: moses1@1lnl.gov that scale to ignition conditions. Of particular importance is the demonstration of peak hohlraum temperatures near $300 \mathrm{eV}$ with overall backscatter less than $10 \%$. Cryogenic target capability and additional diagnostics are being installed in preparation for layered target deuterium-tritium implosions to be conducted later in 2010. Important national security and basic science experiments have also been conducted on NIF. This paper describes the unprecedented experimental capabilities of NIF and the results achieved so far on the path toward ignition, for stockpile stewardship, and the beginning of frontier science experiments. The paper will also address our plans to transition NIF to a national user facility, providing access to NIF for researchers from the DOE laboratories, as well as the national and international academic and fusion energy communities.

Keywords National Ignition Facility · National Ignition Campaign · Inertial fusion energy · Inertial confinement fusion · High energy density science $\cdot$ Laser inertial fusion energy

\section{The National Ignition Facility}

The National Ignition Facility (NIF) is the U.S. Department of Energy (DOE) and National Nuclear Security Administration (NNSA) center to study inertial confinement fusion (ICF) and high energy density (HED) science (Moses 2008a, 2008b). NIF is by far the largest scientific project ever successfully completed by the DOE (LLNL 2005). The 192-beam football stadium-sized NIF is now operational at Lawrence Livermore National Laboratory (LLNL). A total 192-beam energy of 1.1 MJ of ultraviolet laser light was 
demonstrated on March 10, 2009, over 30 times more energy than ever produced in an ICF laser system.

NIF's 192 beams are directed into a 10-meter-diameter vacuum target chamber containing a $\sim 1$-cm-long cylindrical hohlraum target. The NIF target chamber contains entry ports for all the laser beams and over 100 ports for diagnostic instrumentation and target insertion. Sophisticated diagnostic instruments such as X-ray and neutron spectrometers, X-ray imagers, and streak cameras can be mounted around the equator and at the poles of the target chamber. The laser interaction with the hohlraum will produce a radiation field with temperatures of several hundred $\mathrm{eV}$. The resulting hohlraum conditions will provide the necessary environment to explore a wide range of HED science experiments, including laboratory-scale thermonuclear ignition and burn (Moses and Meier 2008).

NIF is also the largest optical instrument ever constructed, with over 38,000 large and small optics and 60,000 points controlled by two million lines of software. The NIF ultraviolet energy specification of $1.8 \mathrm{MJ}$ requires an order of magnitude increase in operating fluence over previous laser systems. Developing high quality optics that can withstand the NIF environment has been a major research and development focus at LLNL. A systematic and robust approach for optics finishing and maintenance has been developed to support the demanding requirements for ignition.

\section{The National Ignition Campaign}

The NIF ignition program is being executed via the National Ignition Campaign (NIC), a national effort that includes General Atomics (GA), LLNL, Los Alamos National Laboratory (LANL), Sandia National Laboratories (SNL), the University of Rochester Laboratory for Laser Energetics (LLE), and a variety of national and international collaborators. The NIC has two major goals: beginning ignition experiments with cryogenic, layered ignition targets in late 2010, and demonstration of a reliable and repeatable ignition platform by the conclusion of NIC at the end of FY2012. The scope for NIC includes the ignition physics program as well as the development of the diagnostics, targets, target cryogenic system, phase plates and other optics, and personnel and environmental protection activities required to execute ignition experiments. The NIC will also develop the infrastructure and processes required to operate NIF as a national user facility.

NIF ignition experiments use a centimeter-scale hohlraum containing a 2-millimeter-scale thin-walled plastic or beryllium capsule filled with a mixture of deuterium and tritium (Lindl 1998; Lindl et al. 2004). Compression of the capsule by a $>280-\mathrm{eV}$ radiation field in the ignition hohlraum drives the DT fuel to conditions under which it will ignite and burn, liberating more energy than is required to initiate the fusion reaction. NIF is designed to achieve target temperatures of 100 million $\mathrm{K}$, radiation temperatures over 3.5 million $\mathrm{K}$, density of $1,000 \mathrm{~g} / \mathrm{cm}^{3}$ and 100 billion times atmospheric pressure. These conditions have never been created before in a laboratory and exist naturally only in the interiors of planetary and stellar environments.

The campaign to initially demonstrate NIF ignition is proceeding in four phases. In the first or "drive" step, the empty hohlraum is tuned to produce the necessary radiation drive on the capsule as a function of time. In the second step, "capsule tuning," a variety of non-cryogenic and cryogenic deuterium-filled capsules are used to adjust the hohlraum symmetry, shock timing, velocity and mass ablated so as to produce the conditions in the imploding capsule required for ignition when a cryogenic fuel layer is incorporated in follow-on experiments. The third step consists of layered cryogenic implosions conducted with a nominal $74 \% / 24 \% / 2 \%$ mixture of tritium, hydrogen, and deuterium (THD) respectively. The reduced yield from these THD targets allows the full diagnostic suite to be employed and the presence of the required pre-burn temperature and fuel areal density to be verified. The final step is DT ignition implosions with expected gains of 10-20. The DT ignition experiments will be conducted with $E_{\text {laser }} \sim 1.3 \mathrm{MJ}$. Laser energies of up to $1.8 \mathrm{MJ}$ should be available for subsequent experiments.

Since August 2009, the NIF team has been conducting experiments in support of the NIC focused on the first or "drive" step discussed above. These initial experiments were aimed at understanding the energetics of the NIF ignition hohlraum and initial capsule-tuning experiments. The results from these initial experiments show promise for achievement of ignition. Capsule implosion experiments at energies up to $1.2 \mathrm{MJ}$ have demonstrated laser energy absorption, radiation temperatures, and symmetry control that scale to ignition conditions. Of particular importance is the demonstration of peak hohlraum temperatures near $300 \mathrm{eV}$, with overall backscatter of less than $10 \%$. Cryogenic target capability and additional diagnostics are being installed in preparation for the beginning of layered cryogenic THD implosions to be conducted later in 2010.

Early results from the 2009 campaign, using hohlraums filled with $\mathrm{He} / \mathrm{H}$ mixtures, indicated that the capsule was compressed into a pancake shape, rather than a uniformly round sphere (see Fig. 1). That is, less energy reached the equatorial regions of the pellet than the top and bottom, likely because light was scattering within the hohlraum (Meezan et al. 2010). The inner cone needed more power to make up for scattering losses and avoid a pancake shape. Shifting the power balance of the two cones required changing the wavelength of beams forming the outer cone by a few angstroms. 

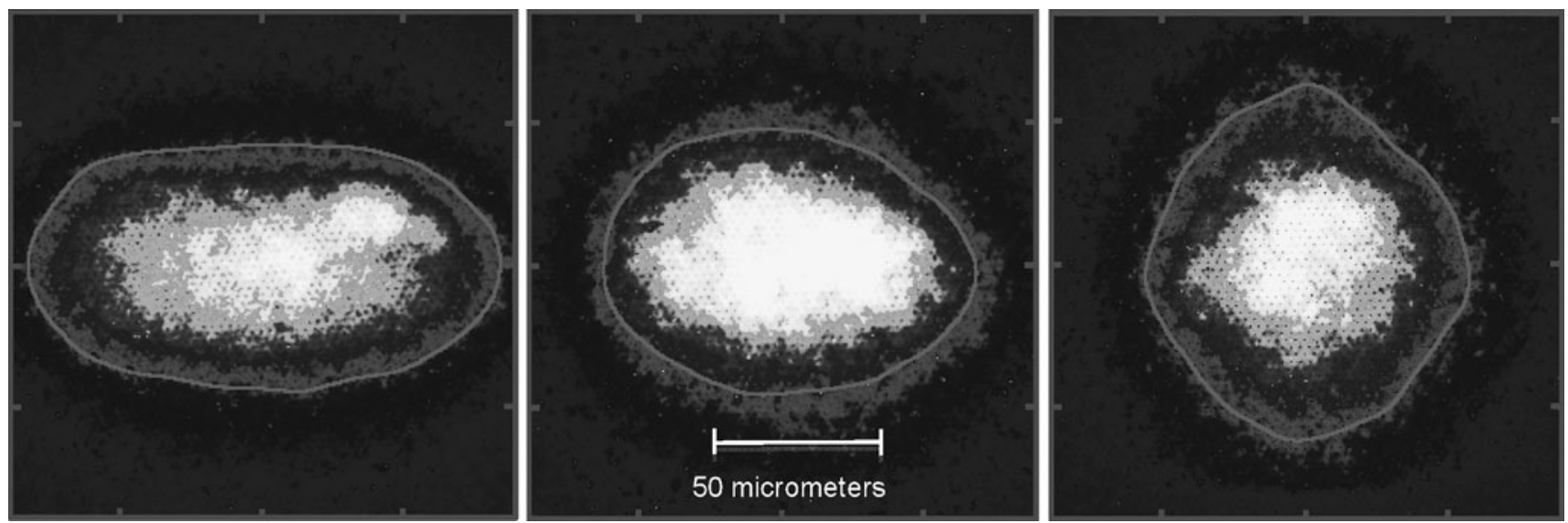

Fig. 1 X-ray emission images from a NIF shot demonstrate how wavelength tuning transforms compression, changing the fuel capsule from a pancake shape to a sphere that is much more symmetric

This new technique, called wavelength tuning, uses the grating effect that occurs when overlapping beams, forming the inner and outer cones, enter the hohlraum and interact with the plasma they create (see Fig. 2). Tuning these beams with respect to one another controls the power distribution in the hohlraum (Michel et al. 2009a, 2009b). This is achieved by shifting the wavelength of some of the laser beams, which detunes the transfer process and controls the energy transfer between the crossing laser beams. With this technique, the laser can deliver more or less energy to different parts of the hohlraum without having to rebalance the energy of the laser beams. This sort of precision tuning is possible because of NIF's flexibility and allows shot designers to make the most efficient use of the available beam energy.

Currently, ignition diagnostics and the cryogenic layering system for ignition targets are being installed on NIF. Initial experiments with layered targets will begin in September 2010 using an ultraviolet laser energy of approximately 1.3 MJ. This energy is consistent with the lasercommissioning plan that ramps up to the planned 192beam operating energy of $1.8 \mathrm{MJ}$ by late 2011 . Initial experiments will be executed with a capsule ablator consisting of a plastic $(\mathrm{CH})$ shell with a Ge dopant. The capsule is filled with fusion fuel (T, H, and D) via a 10$\mu$ m-diameter $\mathrm{SiO}_{2}$ fill tube. The beta decay of $\mathrm{T}$ coupled with precise control of the temperature in the hohlraum is used to generate a high-quality layer of solid THD with a residual gas fill. The Ge dopant density is varied radially through the ablator with a peak concentration of $\sim 1 \%$ near the inner surface of the ablator. Capsule designs using a $\mathrm{Be}$ ablator doped with $\mathrm{Cu}$ are also under consideration. Maintaining these two design options reduces risk and allows the choice of a target that is optimally configured to the precise laser and target fabrication capabilities available.
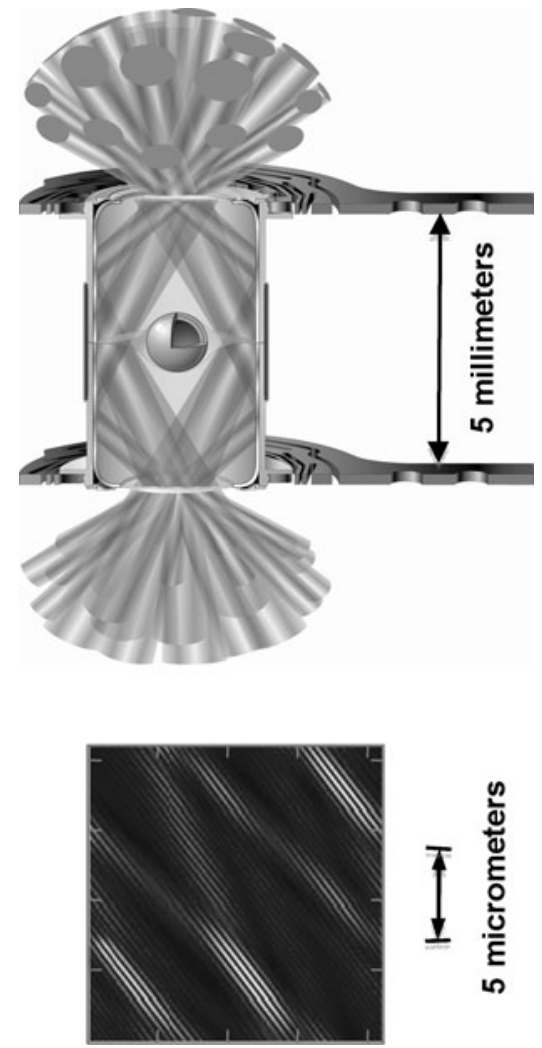

Fig. 2 (Top) NIF's 192 laser beams enter holes at the top and bottom of the hohlraum and strike its inner walls, forming an X-ray field that heats the fuel capsule at the center. (Bottom) Results from a NIF experiment show the laser beams intersecting at a hohlraum entrance hole. This region is a key area in tuning laser light to achieve symmetric compression

Experiments at other laser facilities such as OMEGA at LLE, Trident at LANL and Jupiter at LLNL have been and continue to be used to develop and demonstrate symmetry tuning, shock timing, laser ablation and the diagnostics needed to achieve ignition. 


\section{NIF: a platform for HED science}

While the ignition experiments will be the primary focus through 2012, NIF is executing other experiments in support of stockpile stewardship, national security, and fundamental HED science (Moses 2008c). As mentioned above, NIF is uniquely capable of creating the conditions found in the interiors of stars and deep inside planets. Accompanied by a suite of precise diagnostic equipment, NIF is poised to investigate HED conditions relevant to understanding astrophysical phenomena such as star formation, the makeup of stellar and planetary interiors, the composition of stellar atmospheres, radiation-driven molecular clouds, gamma-ray bursts, and supernovae.

NIF experiments will allow the study of physical processes at temperatures approaching 100 million $\mathrm{K}$, radiation temperatures of more than 3.5 million $\mathrm{K}$, densities greater than $1000 \mathrm{~g} / \mathrm{cm}^{3}$ and pressures of more than 100 billion atmospheres. These conditions exist naturally only during thermonuclear burn, in supernovae and in the fusion reactions that power our stars. A variety of experiments will also be conducted to explore matter at extreme conditions, including studies of material properties, hydrodynamics, and the interaction of intense radiation fields with matter.

NIF experiments are typically executed via experimental "platforms," which consist of the integration of the laser, a target that is most often a hohlraum, and a diagnostic suite capable of providing well-characterized pressure, temperature, implosion, or other environments. Platforms, once developed, can be used for a variety of purposes.

Currently, several platforms are being developed for NIF users. These platforms are designed to study: radiation transport, material properties, hydrodynamics, hohlraum energetics, X-ray sources, shock timing, streaked radiography, capsule implosions and X-ray opacity. Below are descriptions of three of these platforms. Additional information on these NIF experimental platforms and discussion of general NIF capabilities can be found at the NIF website (LLNL 2009).

- Radiation transport. The first experimental platform to be commissioned will provide new capabilities to study the interaction of an intense flux of radiation with matter in planar geometry. The goal of this effort is to commission capabilities for radiation transport experiments, which include the generation of intense radiation flux, the fabrication and characterization of the complex targets needed, a suite of diagnostics to characterize the radiation and subsequent response of the matter driven by the radiation, and data analysis methods. With minor modifications of these four proficiencies, a wide variety of experiments can be carried out without having to develop a new set of capabilities for each experiment. These include investigations into the intriguing Eagle Nebula Pillars 6,500 light years away, thought to be the birthplace of millions of stars; the formation of Herbig-Haro jets, small patches of nebulous clouds ejected when young stars collide at high speeds with nearby clouds of gas and dust; and non-linear ablation front hydrodynamics-physical instabilities that could limit the performance of targets in ICF implosions.

- Material properties. The next experimental platform to be developed on NIF will be for the study of highpressure material properties, creating conditions comparable to planetary interiors. This will allow generation of data to compare with material models and provide better understanding of the make-up of planets. The NIF highpressure materials platform will be able to reach conditions in iron up to 20 megabars, or 20 million times the earth's atmosphere. Experiments at pressures up to 30 megabars in carbon are also planned. With this new data, models can be validated or changed in ways that may alter our understanding about how planets were created or how molten materials behave at extreme pressures and temperatures.

- Hydrodynamics. Following the materials platform, a hydrodynamics platform will be developed to enable the study of fundamental hydrodynamics and instabilities. The processes of interest include compressible mixing, strong shock phenomena, radiative shocks, high Mach number jets, compressible shear flow, and instabilities (e.g., compressible Rayleigh-Taylor, RichtmyerMeshkov, Kelvin-Helmholtz and Weibel). These processes are also present in astrophysical phenomena, such as supernovae, supernovae remnants, and star formation via shocks traveling through molecular clouds. Scaled experiments on NIF can simulate complex flows in the laboratory to compare with models of astrophysical phenomena. NIF will be able to drive high Mach number and compressible flows, both in the presence and absence of radiation, in planar, convergent and divergent geometry. This flexibility allows exploring models and simulations in regimes otherwise not possible in a laboratory.

Also under development are platforms that would produce information about the nuclear reactions relevant to the stars. Early effort will focus on neutron capture reactions relevant to the astrophysical s-process, or "slow-neutron-captureprocess," by which half of the nuclei heavier than iron are synthesized in stars. Another platform that is under consideration will enable detection of charged particles from the reactions that occur in the NIF targets. This platform will enable study of reactions that occur in stars. This will produce data that do not have to be corrected from the "cold target" conditions in which they are usually measured to the high-temperature and density plasma conditions that exist in stars.

NIF will ultimately be a major international center for fundamental HED science. In 2009, a call for proposals was issued with two components: (1) requests for facility time 
and (2) proposals for concept development. The response from the broad national and international communities has been outstanding with 44 letters of intent for facility time and 41 concept development proposals. A peer review committee has been formed to evaluate the facility time proposals and provide recommendations regarding the fiscal year 2011-2012 allocations. Concept development is intended to provide small awards (maximum of $\$ 100 \mathrm{~K}$ ) for those interested in developing experiments on NIF.

\section{Conclusions}

NIF is now operational, with ignition experiments expected to begin in September 2010. After many years of R\&D, most of the pieces needed for demonstrating ignition at NIF are in place, including the NIF laser, a detailed point design with adequate margin, high-quality targets meeting all specifications, and advanced diagnostics capable of precision tuning of capsule performance to ignition conditions. NIF ignition will allow access to the burning plasma regime for the first time, enabling important stockpile stewardship studies and demonstrating the scientific feasibility of ICF. More generally, NIF's ability to create extraordinarily high pressures, temperatures, and densities-as much as 1 trillion atmospheres of pressure, 100 million $\mathrm{K}$ temperature, and $1,000 \mathrm{~g} / \mathrm{cm}^{3}$ density-will enable major fundamental advances in support of DOE's national security, energy, and fundamental science missions. NIF and other major facilities worldwide will launch a new era in HED science, and the demonstration of ignition may one day lead to an inexhaustible clean power supply for humanity.

Ignition at NIF will be a landmark scientific achievement and will open new possibilities for clean, sustainable energy production (Moses 2008d). NIF will also allow fundamental study of matter at extreme energy densities, including the possibility of examining many astrophysical phenomena in the laboratory for the first time. NIF will open a truly new era in HED science.

Acknowledgements This work performed under the auspices of the U.S. Department of Energy by Lawrence Livermore National Laboratory under Contract DE-AC52-07NA27344. LLNL-JRNL-432971.
Open Access This article is distributed under the terms of the Creative Commons Attribution Noncommercial License which permits any noncommercial use, distribution, and reproduction in any medium, provided the original author(s) and source are credited.

\section{References}

Lindl, J.D.: Inertial Confinement Fusion: The Quest for Ignition and Energy Gain Using Indirect Drive. AIP, New York (1998)

Lindl, J.D., Amendt, P., Berger, R.L., Glendinning, S.G., Glenzer, S.H., Haan, S.W., Kauffman, R.L., Landen, O.L., Suter, L.J.: The physics basis for ignition using indirect drive targets on the NIF. Phys. Plasmas 11(2), 339-491 (2004)

LLNL report: National Ignition Campaign Execution Plan, UCRL-AR213718, NIF-0111975-AA (2005)

LLNL web site: http://lasers.llnl.gov/for_users (2009)

Meezan, N.B., Atherton, L.J., Callahan, D.A., Dewald, E.L., Dixit, S., Dzenitis, E.G., Edwards, M.J., Haynam, C.A., Hinkel, D.E., Jones, O.S., Landen, O., London, R.A., Michel, P.A., Moody, J.D., Milovich, J.L., Schneider, M.B., Thomas, C.A., Town, R.P.J., Warrick, A.L., Weber, S.V., Widmann, K., Glenzer, S.H., Suter, L.J., MacGowan, B.J., Kline, J.L., Kyrala, G.A., Nikroo, A.: National Ignition Campaign hohlraum energetics. Phys. Plasmas 17, 056304 (2010)

Michel, P., Divol, L., Williams, E.A., Weber, S., Thomas, C.A., Callahan, D.A., Haan, S.W., Salmonson, J.D., Dixit, S., Hinkel, D.E., Edwards, M.J., MacGowan, B.J., Lindl, J.D., Glenzer, S.H., Suter, L.J.: Tuning the implosion symmetry of ICF targets via controlled crossed-beam energy transfer. Phys. Rev. Lett. 102, 025004 (2009a)

Michel, P., Divol, L., Williams, E.A., Thomas, C.A., Callahan, D.A., Weber, S., Haan, S.W., Salmonson, J.D., Meezan, N.B., Landen, O.L., Dixit, S., Hinkel, D.E., Edwards, M.J., MacGowan, B.J., Lindl, J.D., Glenzer, S.H., Suter, L.J.: Energy transfer between laser beams crossing in ignition hohlraums. Phys. Plasmas 16, 042702 (2009b)

Moses, E.I.: Overview of the National Ignition Facility. Fus. Sci. Technol. 54(2), 361-366 (2008a)

Moses, E.I.: Multi-megajoule NIF: Ushering in a new era in high energy density science. Proc. SPIE Int. Soc. Opt. Eng. 7005, 70050F (2008b)

Moses, E.I.: Ignition on the National Ignition Facility. J. Phys., Conf. Ser. 112(1), 012003 (2008c)

Moses, E.I.: The National Ignition Facility (NIF): a path to fusion energy. Energy Convers. Manag. 49(7), 1795-1802 (2008d)

Moses, E.I., Meier, W.R.: The National Ignition Facility and the golden age of high energy density science. IEEE Trans. Plasma Sci. 36(3), 802-808 (2008) 